Proceedings of the Edinburgh Mathematical Society (2003) 46, 269-277 (C)

DOI:10.1017/S0013091502000214 Printed in the United Kingdom

\title{
HILBERT MODULAR PSEUDODIFFERENTIAL OPERATORS OF MIXED WEIGHT
}

\author{
MIN HO LEE ${ }^{1}$ AND HYO CHUL MYUNG ${ }^{2}$ \\ ${ }^{1}$ Department of Mathematics, University of Northern Iowa, \\ Cedar Falls, IA 50614, USA (lee@math.uni.edu) \\ ${ }^{2}$ Korea Institute for Advanced Study, 20\%-43 Chunryangri-dong, \\ Dongdaemoon-ku, Seoul 130-012, Korea (hm@kias.re.kr)
}

(Received 20 February 2002)

\begin{abstract}
We introduce an action of a discrete subgroup $\Gamma$ of $S L(2, \mathbb{R})^{n}$ on the space of pseudodifferential operators of $n$ variables, and construct a map from the space of Hilbert modular forms for $\Gamma$ to the space of pseudodifferential operators invariant under such a $\Gamma$-action, which is a lifting of the symbol map of pseudodifferential operators. We also obtain a necessary and sufficient condition for a certain type of pseudodifferential operator to be $\Gamma$-invariant.
\end{abstract}

Keywords: Hilbert modular forms; pseudodifferential operators; automorphic forms

2000 Mathematics subject classification: Primary 11F41; 35S05

\section{Introduction}

Pseudodifferential operators are formal Laurent series in the formal inverse $\partial^{-1}$ of the differential operator $\partial=\mathrm{d} / \mathrm{d} z$ on $\mathbb{C}$ whose coefficients are functions on $\mathbb{C}$, and they have been studied in connection with various topics in mathematics. One of the most celebrated applications of pseudodifferential operators in recent years occurs in the theory of soliton equations, which are a certain class of nonlinear integrable partial differential equations including many well-known equations in mathematical physics. Our interest in this paper centres around connections between automorphic forms and pseudodifferential operators defined on the Poincaré upper half-plane $\mathcal{H} \subset \mathbb{C}$, which have been explored recently by Cohen, Manin and Zagier in [1] (see also [4]).

Let $\Gamma$ be a discrete subgroup of $S L(2, \mathbb{R})$, and consider integers $\kappa_{1}$ and $\kappa_{2}$. If $\psi$ is a pseudodifferential operator, we set

$$
\left(\left.\psi\right|_{\kappa_{1}, \kappa_{2}} \gamma\right)(z)=(c z+d)^{-\kappa_{1}} \psi\left(\frac{a z+b}{c z+d}\right)(c z+d)^{\kappa_{2}}
$$

for all $z \in \mathcal{H}$ and $\gamma=\left(\begin{array}{ll}a & b \\ c & d\end{array}\right) \in \Gamma$. Then the map $\left.\psi \mapsto \psi\right|_{\kappa_{1}, \kappa_{2}} \gamma$ for $\gamma \in \Gamma$ determines an action of $\Gamma$ on the space of pseudodifferential operators, and an invariant element under this action is an automorphic pseudodifferential operator of mixed weight $\left(\kappa_{1}, \kappa_{2}\right)$. If 
such an automorphic pseudodifferential operator is of the form $\psi(z)=\sum_{n \geqslant 0} f_{n}(z) \partial^{w-n}$ with $w \in \mathbb{Z}$, then its leading coefficient $f_{0}$ of $\psi$ is an automorphic form for $\Gamma$ of weight $\kappa_{1}-\kappa_{2}-2 w$. Thus there is a natural linear map from the space of $\Gamma$-invariant pseudodifferential operators to the space of automorphic forms for $\Gamma$, and it was Cohen, Manin and Zagier [1] who constructed a lifting of such a map. Since the product of two $\Gamma$-invariant pseudodifferential operators is $\Gamma$-invariant, such a lifting determines a non-commutative multiplication operation on the space of automorphic forms for $\Gamma$. This operation can in fact be expressed in terms of Rankin-Cohen brackets (see [4]), which are bilinear operators on the space of automorphic forms given by certain bilinear combinations of derivatives.

The task of generalizing the theory of pseudodifferential operators to the case of more than one variable was initiated only recently by Parshin in [3]. He introduced pseudodifferential operators of several variables and studied their Poisson structures as well as their connections with soliton equations. It would be natural to expect connections of such pseudodifferential operators with automorphic forms of several variables, that is, Hilbert modular forms. In this paper, we investigate such connections by extending the results of Cohen, Manin and Zagier described above to the case of several variables. In particular, we construct a map from the space of Hilbert modular forms to the space of invariant pseudodifferential operators of several variables, which is a lifting of the symbol map of pseudodifferential operators; this extends one of the results discussed in [2]. We also obtain a necessary and sufficient condition for a certain type of pseudodifferential operator to be $\Gamma$-invariant.

\section{Pseudodifferential operators}

In this section we review the definition of pseudodifferential operators of $n$ variables with $n \geqslant 1$ introduced by Parshin $[\mathbf{3}]$ and describe some of their properties. Let $\left(z_{1}, \ldots, z_{n}\right)$ be the standard coordinate system for $\mathbb{C}^{n}$, and let $\partial_{1}, \ldots, \partial_{n}$ be the differential operators given by

$$
\partial_{1}=\frac{\partial}{\partial z_{1}}, \ldots, \partial_{n}=\frac{\partial}{\partial z_{n}} .
$$

Let $\mathcal{H}^{n} \subset \mathbb{C}^{n}$ be the product of $n$ copies of the Poincaré upper half-plane $\mathcal{H} \subset \mathbb{C}$, and let $\mathcal{F}$ be the ring of holomorphic functions $f(z)=f\left(z_{1}, \ldots, z_{n}\right)$ on $\mathcal{H}^{n}$.

For convenience we adopt the multi-index notation throughout the paper. Thus, given $\alpha=\left(\alpha_{1}, \ldots, \alpha_{n}\right) \in \mathbb{Z}^{n}$ and $u=\left(u_{1}, \ldots, u_{n}\right) \in \mathbb{C}^{n}$, we have

$$
\partial^{\alpha}=\partial_{1}^{\alpha_{1}} \cdots \partial_{n}^{\alpha_{n}}, \quad u^{\alpha}=u_{1}^{\alpha_{1}} \cdots u_{n}^{\alpha_{n}}, \quad|\alpha|=\alpha_{1}+\cdots+\alpha_{n} .
$$

If $\beta=\left(\beta_{1}, \ldots, \beta_{n}\right) \in \mathbb{Z}_{+}^{n}$ with $\mathbb{Z}_{+}^{n}$ denoting the set of non-negative integers, we write $\beta !=\beta_{1} ! \cdots \beta_{n} !$ and

$$
\left(\begin{array}{c}
\alpha \\
\beta
\end{array}\right)=\left(\begin{array}{c}
\alpha_{1} \\
\beta_{1}
\end{array}\right) \cdots\left(\begin{array}{c}
\alpha_{n} \\
\beta_{n}
\end{array}\right)
$$

where for $1 \leqslant i \leqslant n$ we have $\left(\begin{array}{c}\alpha_{i} \\ 0\end{array}\right)=1$ and

$$
\left(\begin{array}{c}
\alpha_{i} \\
\beta_{i}
\end{array}\right)=\frac{\alpha_{i}\left(\alpha_{i}-1\right) \cdots\left(\alpha_{i}-\beta_{i}+1\right)}{\beta_{i} !}
$$


for $\beta_{i}>0$. Furthermore, for $\mu=\left(\mu_{1}, \ldots, \mu_{n}\right), \nu=\left(\nu_{1}, \ldots, \nu_{n}\right) \in \mathbb{Z}^{n}$ we write $\mu \leqslant \nu$ if $\mu_{i} \leqslant \nu_{i}$ for each $i=1, \ldots, n$, and also write $\boldsymbol{c}=(c, \ldots, c) \in \mathbb{Z}^{n}$ if $c \in \mathbb{Z}$.

Definition 2.1. A pseudodifferential operator of $n$ variables is a formal series of the form

$$
L=\sum_{\alpha \leqslant \nu} f_{\alpha}(z) \partial^{\alpha}
$$

for some $\nu \in \mathbb{Z}^{n}$, where $z=\left(z_{1}, \ldots, z_{n}\right) \in \mathcal{H}^{n}$ and $f_{\alpha} \in \mathcal{F}$ for all $\alpha \in \mathbb{Z}^{n}$ with $\alpha \leqslant \nu$. We shall denote by $\Psi D O$ the complex vector space consisting of all pseudodifferential operators of $n$ variables.

We now introduce the notion of order for pseudodifferential operators of several variables. Although other definitions are possible, we shall employ the one used by Parshin in $[3]$.

\section{Definition 2.2.}

(i) The order $\operatorname{ord}(L)$ of an element $L \in \Psi \mathrm{DO}$ is the smallest integer $r$ such that $L=\sum_{i \leqslant r} a_{i} \partial_{n}^{i}$ with $a_{r} \neq 0$.

(ii) The highest term $\operatorname{HT}(L)$ of $L \in \Psi \mathrm{DO}$ is the term in $L$ defined inductively by

$$
\operatorname{HT}(L)=\left(\operatorname{HT}\left(a_{r}\right)\right) \partial_{n}^{r}
$$

for $L=\sum_{i \leqslant r} a_{i} \partial_{n}^{i}$ with $\operatorname{ord}(L)=r$.

If the highest term of $L$ is of the form $\operatorname{HT}(L)=f(z) \partial_{1}^{\eta_{1}} \cdots \partial_{n}^{\eta_{n}}$, then we set

$$
\nu(L)=\left(\eta_{1}, \ldots, \eta_{n}\right) \in \mathbb{Z}^{n} .
$$

Given an element $\omega=\left(\omega_{1}, \ldots, \omega_{n}\right) \in \mathbb{Z}^{n}$, we consider the subspaces $\Psi \mathrm{DO}_{\omega}$ and $\Psi \mathrm{DO}_{\omega}^{*}$ of $\Psi \mathrm{DO}$ defined by

$$
\begin{aligned}
& \Psi \mathrm{DO}_{\omega}=\{L \in \Psi \mathrm{DO} \mid \nu(L) \preceq \omega\}, \\
& \Psi \mathrm{DO}_{\omega}^{*}=\{L \in \Psi \mathrm{DO} \mid \nu(L) \prec \omega\},
\end{aligned}
$$

where ' $\prec$ ' is a lexicographic type of order such that $\nu(L)=\left(\eta_{1}, \ldots, \eta_{n}\right) \prec 0$ if

$$
\eta_{n}<0, \quad \text { or } \eta_{n}=0 \text { and } \eta_{n-1}<0, \quad \text { or } \ldots \text {, etc., }
$$

and ' $\preceq$ ' means ' $\prec$ ' or '='. Then we obtain a short exact sequence

$$
0 \rightarrow \Psi \mathrm{DO}_{\omega}^{*} \rightarrow \Psi \mathrm{DO}_{\omega} \stackrel{\Xi}{\rightarrow} \mathcal{F} \rightarrow 0
$$

where $\Xi$ is the symbol map sending a pseudodifferential $L \in \Psi \mathrm{DO}_{\omega}$ to the coefficient of its highest term, that is, $\Xi(L)=f_{\omega}(z)$ if $\operatorname{HT}(L)=f_{\omega}(z) \partial^{\omega}$. 
We now consider the action of $S L(2, \mathbb{R})^{n}$ on $\mathcal{H}^{n}$ induced by the usual action of $S L(2, \mathbb{R})$ on $\mathcal{H}$. Thus, if $\gamma \in S L(2, \mathbb{R})^{n}$ and $z=\left(z_{1}, \ldots, z_{n}\right) \in \mathcal{H}^{n}$ with

$$
\gamma=\left(\gamma_{1}, \ldots, \gamma_{n}\right), \quad \gamma_{i}=\left(\begin{array}{ll}
a_{i} & b_{i} \\
c_{i} & d_{i}
\end{array}\right) \in S L(2, \mathbb{R}) \quad(1 \leqslant i \leqslant n),
$$

then we have

$$
\gamma z=\left(\gamma_{1} z_{1}, \ldots, \gamma_{n} z_{n}\right)=\left(\frac{a_{1} z_{1}+b_{1}}{c_{1} z_{1}+d_{1}}, \ldots, \frac{a_{n} z_{n}+b_{n}}{c_{n} z_{n}+d_{n}}\right)
$$

For such $\gamma$ and $z$ we set

$$
j(\gamma, z)=\left(c_{1} z_{1}+d_{1}, \ldots, c_{n} z_{n}+d_{n}\right) \in \mathbb{C}^{n} .
$$

Under the action of $S L(2, \mathbb{R})^{n}$ on $\mathcal{H}^{n}$ given by $(2.7)$, the differential operator $\partial_{i}^{\alpha_{i}}=$ $\partial^{\alpha_{i}} / \partial z_{i}^{\alpha_{i}}$ for $1 \leqslant i \leqslant n$ with $\alpha_{i} \geqslant 1$ is transformed to the pseudodifferential operator

$$
\tilde{\partial}_{i}^{\alpha_{i}}=\left[\left(c_{i} z_{i}+d_{i}\right)^{2} \partial_{i}\right]^{\alpha_{i}}=\sum_{k=0}^{\infty} k !\left(\begin{array}{c}
\alpha_{i} \\
k
\end{array}\right)\left(\begin{array}{c}
\alpha_{i}-1 \\
k
\end{array}\right) c_{i}^{k}\left(c_{i} z_{i}+d_{i}\right)^{2 \alpha_{i}-k} \partial^{\alpha_{i}-k}
$$

(cf. [1]). Thus the differential operator $\partial^{\alpha}$ in (2.1) is transformed to the pseudodifferential operator of $n$ variables given by

$$
\tilde{\partial}^{\alpha}=\tilde{\partial}_{1}^{\alpha_{1}} \cdots \tilde{\partial}_{n}^{\alpha_{n}}=\sum_{\nu \geqslant 0} \nu !\left(\begin{array}{c}
\alpha \\
\nu
\end{array}\right)\left(\begin{array}{c}
\alpha-1 \\
\nu
\end{array}\right) c^{\nu} j(\gamma, z)^{2 \alpha-\nu} \partial^{\alpha-\nu},
$$

where $\alpha=\left(\alpha_{1}, \ldots, \alpha_{n}\right)$.

\section{Invariant operators}

In this section we introduce an action of a discrete subgroup $\Gamma$ of $S L(2, \mathbb{R})^{n}$ on $\Psi$ DO and discuss connections between Hilbert modular forms for $\Gamma$ and pseudodifferential operators of $n$ variables invariant under such a $\Gamma$-action.

Let $\Gamma$ be a discrete subgroup of $S L(2, \mathbb{R})^{n}$, and let $j: \Gamma \times \mathcal{H}^{n} \rightarrow \mathbb{C}^{n}$ be the map given by $(2.8)$. Then we see that

$$
j\left(\gamma \gamma^{\prime}, z\right)=j\left(\gamma, \gamma^{\prime} z\right) j\left(\gamma^{\prime}, z\right)
$$

for all $\gamma, \gamma^{\prime} \in \Gamma$ and $z \in \mathcal{H}^{n}$. If $\eta=\left(\eta_{1}, \ldots, \eta_{n}\right) \in \mathbb{Z}^{n}, \gamma \in \Gamma$, and $f \in \mathcal{F}$, then we define the function $\left.f\right|_{\eta} \gamma: \mathcal{H}^{n} \rightarrow \mathbb{C}$ by

$$
\left(\left.f\right|_{\eta} \gamma\right)(z)=j(\gamma, z)^{-\eta} f(\gamma z)
$$

for all $z \in \mathcal{H}^{n}$. 
Definition 3.1. Given an element $\omega \in \mathbb{Z}^{n}$, a Hilbert modular form of weight $\omega$ for $\Gamma$ is a holomorphic function $f: \mathcal{H}^{n} \rightarrow \mathbb{C}$ such that

$$
\left.f\right|_{\omega} \gamma=f
$$

for all $\gamma \in \Gamma$. We shall denote by $\mathcal{M}_{\omega}(\Gamma)$ the space of all Hilbert modular forms of weight $\omega$ for $\Gamma$.

If $\xi, \eta \in \mathbb{Z}^{n}, \gamma \in \Gamma$ and $L \in \Psi$ DO, we define the associated pseudodifferential operator $\left.L\right|_{\xi, \eta} \gamma$ by

$$
\left(\left.L\right|_{\xi, \eta} \gamma\right)(z)=j(\gamma, z)^{-\xi} L(\gamma z) j(\gamma, z)^{\eta}
$$

for all $z \in \mathcal{H}^{n}$. Then, using (3.1), we see that the map $\left.L \mapsto L\right|_{\xi, \eta} \gamma$ determines an action of $\Gamma$ on $\Psi$ DO.

Definition 3.2. Given $\xi, \eta \in \mathbb{Z}^{n}$, a Hilbert modular pseudodifferential operator of mixed weight $(\xi, \eta)$ for $\Gamma$ is an element of $\Psi \mathrm{DO}$ that is invariant under the $\Gamma$-action given by (3.2). We denote by $\Psi \mathrm{DO}^{\xi, \eta}$ the complex vector space consisting of all Hilbert modular pseudodifferential operators of mixed weight $(\xi, \eta)$ for $\Gamma$.

If $f \in \mathcal{M}_{2 \alpha}(\Gamma)$ and $g \in \mathcal{M}_{2 \beta}(\Gamma)$ for some $\alpha, \beta \in \mathbb{Z}_{+}^{n}$, we set

$$
[f, g]_{\nu}^{(\alpha, \beta)}(z)=\sum_{0 \leqslant \lambda \leqslant \nu}(-1)^{|\lambda|}\left(\begin{array}{c}
2 \alpha+\nu-\mathbf{1} \\
\nu-\lambda
\end{array}\right)\left(\begin{array}{c}
2 \beta+\nu-\mathbf{1} \\
\lambda
\end{array}\right)\left(\partial^{\lambda} f(z)\right)\left(\partial^{\nu-\lambda} g(z)\right)
$$

for all $z \in \mathcal{H}^{n}$ and $\nu \in \mathbb{Z}_{+}^{n}$. Then the function $[f, g]_{\nu}^{(\alpha, \beta)}: \mathcal{H}^{n} \rightarrow \mathbb{C}$ given by (3.3) is a Hilbert modular form of weight $2 \alpha+2 \beta+2 \nu$ for $\Gamma$ (see [2, Theorem 5.1]), and the bilinear map $(f, g) \mapsto[f, g]_{\nu}^{(\alpha, \beta)}$ on $\mathcal{M}_{2 \alpha}(\Gamma) \times \mathcal{M}_{2 \beta}(\Gamma)$ is the Hilbert modular version of the Rankin-Cohen bracket described in [1] and [4]. Given $\xi, \eta, \omega \in \mathbb{Z}^{n}$, we define the linear map $\mathcal{D}_{\omega}^{\xi, \eta}: \mathcal{F} \rightarrow \Psi \mathrm{DO}_{\omega}$ by

$$
\mathcal{D}_{\omega}^{\xi, \eta}(f)=\sum_{\mu \geqslant 0} \frac{\left(\begin{array}{c}
\omega \\
\mu
\end{array}\right)\left(\begin{array}{c}
\omega+\eta-\mathbf{1} \\
\mu
\end{array}\right)}{\left(\begin{array}{c}
\eta-\xi+2 \omega \\
\mu
\end{array}\right)}\left(\partial^{\mu} f\right) \partial^{\omega-\mu}
$$

for all $f \in \mathcal{F}$.

Proposition 3.3. Let $f \in \mathcal{M}_{2 \alpha}(\Gamma)$ and $g \in \mathcal{M}_{2 \beta}(\Gamma)$ for some $\alpha, \beta \in \mathbb{Z}^{n}$. Then we have

$$
\left(\mathcal{D}_{\nu}^{2 \alpha+2 \beta+2 \nu, 2 \beta} f\right) g=\left(\begin{array}{c}
2 \alpha+\nu-\mathbf{1} \\
\nu
\end{array}\right)^{-1}[f, g]_{\nu}^{(\alpha, \beta)}
$$

for all $\nu \in \mathbb{Z}_{+}^{n}$.

Proof. Given $\nu \in \mathbb{Z}_{+}^{n}$, by (3.4) we have

$$
\left(\mathcal{D}_{\nu}^{2 \alpha+2 \beta+2 \nu, 2 \beta} f\right) g=\sum_{\lambda \geqslant 0} \frac{\left(\begin{array}{l}
\nu \\
\lambda
\end{array}\right)\left(\begin{array}{c}
\nu+2 \beta-\mathbf{1} \\
\lambda
\end{array}\right)}{\left(\begin{array}{c}
-2 \alpha \\
\lambda
\end{array}\right)}\left(\partial^{\lambda} f\right)\left(\partial^{\nu-\lambda} g\right) .
$$


Since $[f, g]_{\nu}^{(\alpha, \beta)}$ is given by (3.3), in order to prove (3.5) it suffices to show that

$$
(-1)^{|\lambda|}\left(\begin{array}{c}
-2 \alpha \\
\lambda
\end{array}\right)\left(\begin{array}{c}
2 \alpha+\nu-\mathbf{1} \\
\nu-\lambda
\end{array}\right)=\left(\begin{array}{c}
\nu \\
\lambda
\end{array}\right)\left(\begin{array}{c}
2 \alpha+\nu-\mathbf{1} \\
\nu
\end{array}\right)
$$

However, we see that

$$
\begin{aligned}
(-1)^{|\lambda|}\left(\begin{array}{c}
-2 \alpha \\
\lambda
\end{array}\right) & \left(\begin{array}{c}
2 \alpha+\nu-\mathbf{1} \\
\nu-\lambda
\end{array}\right) \\
& =(-1)^{|\lambda|} \prod_{i=1}^{n} \frac{\left(-2 \alpha_{i}\right) \cdots\left(-2 \alpha_{i}-\lambda_{i}+1\right)}{\lambda_{i} !} \prod_{i=1}^{n} \frac{\left(2 \alpha_{i}+\nu_{i}-1\right) \cdots\left(2 \alpha_{i}+\lambda_{i}\right)}{\left(\nu_{i}-\lambda_{i}\right) !} \\
& =\prod_{i=1}^{n} \frac{\left(2 \alpha_{i}+\nu_{i}-1\right) \cdots\left(2 \alpha_{i}\right)}{\lambda_{i} !\left(\nu_{i}-\lambda_{i}\right) !} \\
& =\prod_{i=1}^{n}\left(\begin{array}{c}
\nu_{i} \\
\lambda_{i}
\end{array}\right)\left(\begin{array}{c}
2 \alpha_{i}+\nu_{i}-1 \\
\nu_{i}
\end{array}\right) \\
& =\left(\begin{array}{c}
\nu \\
\lambda
\end{array}\right)\left(\begin{array}{c}
2 \alpha+\nu-1 \\
\nu
\end{array}\right),
\end{aligned}
$$

and therefore the proposition follows.

Theorem 3.4. Let $\xi, \eta, \omega \in \mathbb{Z}^{n}$, and let $f \in \mathcal{M}_{\xi-\eta-2 \omega}(\Gamma)$. Then $\mathcal{D}_{\omega}^{\xi, \eta} f$ is a Hilbert modular pseudodifferential operator of mixed weight $(\xi, \eta)$ for $\Gamma$, that is, an element of $\Psi \mathrm{DO}^{\xi, \eta}$.

Proof. By (2.9) and (3.2) the condition that

$$
\left.\left(\mathcal{D}_{\omega}^{\xi, \eta} f\right)\right|_{\xi, \eta} \gamma=\mathcal{D}_{\omega}^{\xi, \eta} f
$$

for all $\gamma \in \Gamma, f \in \mathcal{M}_{\xi-\eta-2 \omega}(\Gamma)$ with $\xi, \eta, \omega \in \mathbb{Z}^{n}$ reduces to a finite number of identities of some binomial coefficients involving $\xi, \eta$ and $\omega$. Therefore, in order to prove the theorem, it suffices to verify (3.6) for infinitely many values of $\xi, \eta$ and $\omega$. Thus without losing generality we shall restrict ourselves to the case where $\xi, \eta$ and $\omega$ are of the form

$$
\xi=2 \alpha+2 \beta+2 \nu, \quad \eta=2 \beta, \quad \omega=\nu,
$$

with $\alpha, \beta \in \mathbb{Z}^{n}$ and $\nu \in \mathbb{Z}_{+}^{n}$. If $f \in \mathcal{M}_{2 \alpha}(\Gamma)$ and $g \in \mathcal{M}_{2 \beta}(\Gamma)$, then by (3.5) we see that

$$
\left(\mathcal{D}_{\nu}^{\xi, \eta} f\right) g=\left(\begin{array}{c}
2 \alpha+\nu-1 \\
\nu
\end{array}\right)^{-1}[f, g]_{\nu}^{(\alpha, \beta)} \in \mathcal{M}_{2 \xi}(\Gamma) .
$$

Using this and the fact that $g \in \mathcal{M}_{\eta}(\Gamma)$, we obtain

$$
\begin{aligned}
\left(\left(\left.\left(\mathcal{D}_{\nu}^{\xi, \eta} f\right)\right|_{\xi, \eta} \gamma\right) g\right)(z) & =\left(j(\gamma, z)^{-\xi} \mathcal{D}_{\nu}^{\xi, \eta} f(\gamma z) j(\gamma, z)^{\eta}\right) g(z) \\
& =j(\gamma, z)^{-\xi} \mathcal{D}_{\nu}^{\xi, \eta} f(\gamma z) g(\gamma z) \\
& =j(\gamma, z)^{-\xi}\left(\left(\mathcal{D}_{\nu}^{\xi, \eta} f\right) g\right)(\gamma z) \\
& =\left(\left.\left(\mathcal{D}_{\nu}^{\xi, \eta} f\right) g\right|_{\xi} \gamma\right)(z) \\
& =\left(\left(\mathcal{D}_{\nu}^{\xi, \eta} f\right) g\right)(z)
\end{aligned}
$$


for each $\gamma \in \Gamma$ and $z \in \mathcal{H}^{n}$. Hence it follows that $\mathcal{D}_{\nu}^{\xi, \eta} f$ is an element of $\Psi D O^{\xi, \eta}$, and therefore the proof of the theorem is complete.

For each $\omega \in \mathbb{Z}^{n}$ we now consider the subspaces $\Psi \mathrm{DO}_{\omega}^{\xi, \eta}$ and $\Psi \mathrm{DO}_{\omega^{*}}^{\xi, \eta}$ of $\Psi \mathrm{DO} \mathrm{O}^{\xi, \eta}$ given by

$$
\Psi \mathrm{DO}_{\omega}^{\xi, \eta}=\Psi \mathrm{DO}^{\xi, \eta} \cap \Psi \mathrm{DO}_{\omega}, \quad \Psi \mathrm{DO}_{\omega^{*}}^{\xi, \eta}=\Psi \mathrm{DO}^{\xi, \eta} \cap \Psi \mathrm{DO}_{\omega}^{*},
$$

where $\Psi \mathrm{DO}_{\omega}$ and $\Psi \mathrm{DO}_{\omega}^{*}$ are as in (2.3) and (2.4).

Theorem 3.5. Let $\Gamma$ be a discrete subgroup of $S L(2, \mathbb{R})^{n}$, and let $\mathcal{M}_{\xi-\eta-2 \omega}(\Gamma)$ be the space of Hilbert modular forms of weight $\xi-\eta-2 \omega$ for $\Gamma$ as in Definition 3.1. Then the exact sequence in (2.5) induces the short exact sequence

$$
0 \rightarrow \Psi \mathrm{DO}_{\omega^{*}}^{\xi, \eta} \rightarrow \Psi \mathrm{DO}_{\omega}^{\xi, \eta} \rightarrow \mathcal{M}_{\xi-\eta-2 \omega}(\Gamma) \rightarrow 0
$$

which splits.

Proof. Let $L$ be an element of $\Psi \mathrm{DO}_{\omega}$ whose highest term is $\operatorname{HT}(L)=f_{\omega}(z) \partial^{\omega}$, and let $\tilde{L}$ be the corresponding transformed operator under the action in (3.2) for $\gamma \in \Gamma$. Then the highest term of $\tilde{L}$ is given by

$$
\begin{aligned}
\operatorname{HT}(\tilde{L}(z)) & =\operatorname{HT}\left(j(\gamma, z)^{-\xi} L(\gamma z) j(\gamma, z)^{\eta}\right) \\
& =\operatorname{HT}\left(j(\gamma, z)^{-\xi} j(\gamma, z)^{2 \omega} f_{\omega}(\gamma z) \partial^{\omega} j(\gamma, z)^{\eta}\right) \\
& =j(\gamma, z)^{2 \omega-\xi+\eta} f_{\omega}(\gamma z) \partial^{\omega}
\end{aligned}
$$

for all $z \in \mathcal{H}^{n}$. On the other hand, if $L$ belongs to $\Psi \mathrm{DO}_{\omega}^{\xi, \eta}$, we have

$$
\operatorname{HT}(\tilde{L}(z))=\operatorname{HT}(L(z))=f_{\omega}(z) \partial^{\omega} .
$$

By comparing this with (3.7) we see that $f_{\omega} \in \mathcal{M}_{\xi-\eta-2 \omega}(\Gamma)$. Thus, if $\Xi: \Psi \mathrm{DO}_{\omega}^{\xi, \eta} \rightarrow \mathcal{F}$ is the symbol map in (2.5), we obtain

$$
\Xi\left(\Psi \mathrm{DO}_{\omega}^{\xi, \eta}\right) \subset \mathcal{M}_{\xi-\eta-2 \omega}(\Gamma) .
$$

Since it is clear that

$$
\operatorname{ker} \Xi=\Psi \mathrm{DO}_{\omega^{*}}^{\xi, \eta}
$$

it suffices to show that the sequence splits. For this purpose we consider an element $f \in \mathcal{M}_{\xi-\eta-2 \omega}(\Gamma)$. Then by Theorem 3.4 we see that the pseudodifferential operator $\mathcal{D}_{\omega}^{\xi, \eta} f$ belongs to $\Psi \mathrm{DO}_{\omega}^{\xi, \eta}$. Since $\Xi\left(\mathcal{D}_{\omega}^{\xi, \eta} f\right)=f$, it follows that $D_{\omega}^{\xi, \eta}$ is a lifting of the map $\Xi$, which completes the proof of the theorem.

Remark 3.6. Theorem 3.5 extends Theorem 3.3 in $[\mathbf{2}]$, where the case of $\xi=\eta=\mathbf{0} \in$ $\mathbb{Z}^{n}$ was considered, as well as Proposition 7 in $[\mathbf{1}]$, where the case of $n=1$ was treated. 


\section{Functional equations}

Let $\xi, \eta \in \mathbb{Z}^{n}$, and let $\Gamma$ be a discrete subgroup of $S L(2, \mathbb{R})^{n}$, which acts on the space $\Psi$ DO by (3.2). We denote by $\Psi \mathrm{DO}^{\xi, \eta}$ the subspace of $\Psi \mathrm{DO}$ consisting of $\Gamma$-invariant elements as in Definition 3.2. The next theorem provides a necessary and sufficient condition for a certain type of pseudodifferential operator to be $\Gamma$-invariant.

Theorem 4.1. Let $L$ be an element of $\Psi D O$ given by

$$
L(z)=\sum_{\lambda \geqslant \mathbf{1}}(-1)^{|\lambda|} \lambda !(\lambda-\mathbf{1}) ! h_{\lambda}(z) \partial^{-\lambda}
$$

for some holomorphic functions $h_{\lambda}$ on $\mathcal{H}^{n}$. Then $L$ is an element of $\Psi \mathrm{DO}^{\xi, \eta}$ if and only if

$$
\left(\left.h_{\lambda}\right|_{2 \lambda+\xi-\eta} \gamma\right)(z)=\sum_{\mu=\mathbf{0}}^{\lambda-\mathbf{1}} \sum_{\nu=\mathbf{0}}^{\mu} \frac{(-1)^{|\nu|}(\lambda-\nu) !}{(\mu-\nu) ! \lambda !}\left(\begin{array}{l}
\eta \\
\nu
\end{array}\right) \frac{c^{\mu}}{j(\gamma, z)^{\mu}} h_{\lambda-\mu}(z)
$$

for all $\gamma \in \Gamma$ and $\lambda \geqslant \mathbf{1}$.

Proof. Given $\gamma \in \Gamma$ of the form (2.6), using (3.2), we have

$$
\left(\left.L\right|_{\xi, \eta} \gamma^{-1}\right)(z)=\sum_{\alpha \geqslant \mathbf{1}}(-1)^{|\alpha|} \alpha !(\alpha-\mathbf{1}) ! j\left(\gamma^{-1}, z\right)^{-\xi} h_{\alpha}\left(\gamma^{-1} z\right)\left(j\left(\gamma^{-1}, z\right)^{2} \partial\right)^{-\alpha} j\left(\gamma^{-1}, z\right)^{\eta} .
$$

By using (2.9) we see that

$$
\left(j\left(\gamma^{-1}, z\right)^{-2} \partial\right)^{-\alpha}=\sum_{\beta \geqslant 0} \beta !\left(\begin{array}{c}
-\alpha \\
\beta
\end{array}\right)\left(\begin{array}{c}
-\alpha-\mathbf{1} \\
\beta
\end{array}\right)(-c)^{\beta} j\left(\gamma^{-1}, z\right)^{-2 \alpha-\beta} \partial^{-\alpha-\beta},
$$

where $c=\left(c_{1}, \ldots, c_{n}\right) \in \mathbb{R}^{n}$ is as in (2.6). Using this and the relations

$$
\left(\begin{array}{c}
-\alpha \\
\beta
\end{array}\right)=\frac{(-1)^{|\beta|}(\alpha+\beta-\mathbf{1}) !}{\beta !(\alpha-\mathbf{1}) !}, \quad\left(\begin{array}{c}
-\alpha-\mathbf{1} \\
\beta
\end{array}\right)=\frac{(-1)^{|\beta|}(\alpha+\beta) !}{\beta ! \alpha !},
$$

we obtain

$$
\begin{aligned}
\left(\left.L\right|_{\xi, \eta} \gamma^{-1}\right)(z)=\sum_{\alpha \geqslant \mathbf{1}} \sum_{\beta \geqslant \mathbf{0}} \frac{(-1)^{|\alpha|}}{\beta !}(\alpha+ & \beta-\mathbf{1}) !(\alpha+\beta) !(-c)^{\beta} \\
& \times j\left(\gamma^{-1}, z\right)^{-2 \alpha-\beta-\xi} h_{\alpha}\left(\gamma^{-1} z\right) \partial^{-\alpha-\beta} j\left(\gamma^{-1}, z\right)^{\eta} .
\end{aligned}
$$

On the other hand, by the Leibniz rule the pseudodifferential operator $\partial^{-\alpha-\beta} j\left(\gamma^{-1}, z\right)^{\eta}$ can be written in the form

$$
\begin{aligned}
\partial^{-\alpha-\beta} j\left(\gamma^{-1}, z\right)^{\eta} & =\sum_{\nu \geqslant \mathbf{0}}\left(\begin{array}{c}
-\alpha-\beta \\
\nu
\end{array}\right)\left(\partial^{\nu} j\left(\gamma^{-1}, z\right)^{\eta}\right) \partial^{-\alpha-\beta-\nu} \\
& =\sum_{\nu \geqslant \mathbf{0}}\left(\begin{array}{c}
-\alpha-\beta \\
\nu
\end{array}\right)\left(\begin{array}{l}
\eta \\
\nu
\end{array}\right) \nu !(-c)^{\nu} j\left(\gamma^{-1}, z\right)^{\eta-\nu} \partial^{-\alpha-\beta-\nu}, \\
\left(\begin{array}{c}
-\alpha-\beta \\
\nu
\end{array}\right) & =\frac{(-1)^{|\nu|}(\alpha+\beta+\nu-\mathbf{1}) !}{\nu !(\alpha+\beta-\mathbf{1}) !} .
\end{aligned}
$$


Thus we see that

$$
\begin{aligned}
\left(\left.L\right|_{\xi, \eta} \gamma^{-1}\right)(z)=\sum_{\alpha \geqslant \mathbf{1}} \sum_{\beta \geqslant \mathbf{0}} \sum_{\nu \geqslant \mathbf{0}} \frac{(-1)^{|\alpha+\nu|}}{\beta !}\left(\begin{array}{l}
\eta \\
\nu
\end{array}\right)(\alpha+\beta) !(\alpha+\beta+\nu-\mathbf{1}) ! \\
\quad \times(-c)^{\beta+\nu} h_{\alpha}\left(\gamma^{-1} z\right) j\left(\gamma^{-1}, z\right)^{-2 \alpha-\beta-\nu-\xi+\eta} \partial^{-\alpha-\beta-\nu} .
\end{aligned}
$$

Introducing the indices $\lambda=\alpha+\beta+\nu$ and $\mu=\beta+\nu$, we obtain

$$
\begin{aligned}
\left(\left.L\right|_{\xi, \eta} \gamma^{-1}\right)(z)=\sum_{\lambda \geqslant \mathbf{1}} \sum_{\mu=\mathbf{0}}^{\lambda-1} \sum_{\nu \geqslant \mathbf{0}}^{\mu} \frac{(-1)^{|\lambda+\nu-\mu|}(\lambda-\nu) !(\lambda-\mathbf{1}) !}{(\mu-\nu) !}\left(\begin{array}{l}
\eta \\
\nu
\end{array}\right) \\
\times(-c)^{\mu} h_{\lambda-\mu}\left(\gamma^{-1} z\right) j\left(\gamma^{-1}, z\right)^{-2 \lambda+\mu-\xi+\eta} \partial^{-\lambda} .
\end{aligned}
$$

Since $\left.L\right|_{\xi, \eta} \gamma^{-1}=L$, it follows that

$$
h_{\lambda}(z)=\sum_{\mu=\mathbf{0}}^{\lambda-1} \sum_{\nu \geqslant 0}^{\mu} \frac{(-1)^{|\nu-\mu|}(\lambda-\nu) !}{\lambda !(\mu-\nu) !}\left(\begin{array}{l}
\eta \\
\nu
\end{array}\right)(-c)^{\mu} j\left(\gamma^{-1}, z\right)^{-2 \lambda+\mu-\xi+\eta} h_{\lambda-\mu}\left(\gamma^{-1} z\right) .
$$

Hence, using the relation $j\left(\gamma^{-1}, \gamma z\right)=j(\gamma, z)^{-1}$, we obtain

$$
\begin{aligned}
\left(\left.h_{\lambda}\right|_{2 \lambda+\xi-\eta} \gamma\right)(z) & =h_{\lambda}(\gamma z) j(\gamma, z)^{-2 \lambda-\xi+\eta} \\
& =\sum_{\mu=0}^{\lambda-\mathbf{1}} \sum_{\nu=0}^{\mu} \frac{(-1)^{|\nu|}(\lambda-\nu) !}{(\mu-\nu) ! \lambda !}\left(\begin{array}{l}
\eta \\
\nu
\end{array}\right) \frac{c^{\mu}}{j(\gamma, z)^{\mu}} h_{\lambda-\mu}(z),
\end{aligned}
$$

and therefore the proof of the theorem is complete.

Remark 4.2. If the number of variables $n$ is 1 and if $\xi=\eta=0$, then the result in Theorem 4.1 reduces to the equivalence of (2) and (3) in Proposition 2 in the paper of Cohen, Manin and Zagier [1].

Acknowledgements. H.C.M.'s research was supported by KIAS grant M01004.

\section{References}

1. P. Cohen, Y. Manin and D. Zagier, Automorphic pseudodifferential operators, in Algebraic aspects of nonlinear systems, pp. 17-47 (Birkhäuser, Boston, 1997).

2. M. H. LeE, Hilbert modular pseudodifferential operators, Proc. Am. Math. Soc. 129 (2001), 3151-3160.

3. A. Parshin, On a ring of formal pseudodifferential operators, Proc. Steklov Inst. Math. 224 (1999), 266-280.

4. D. Zagier, Modular forms and differential operators, Proc. Indian Acad. Sci. Math. Sci. 104 (1994), 57-75. 